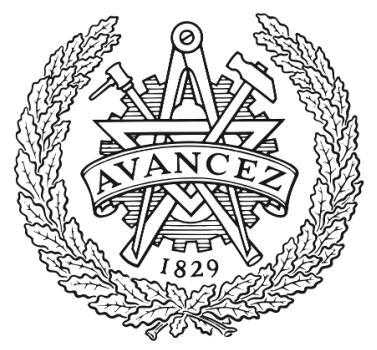

CHALMERS

UNIVERSITY OF TECHNOLOGY

\title{
Hybrid Beamforming Transmitter Modeling for Millimeter-Wave MIMO Applications
}

Downloaded from: https://research.chalmers.se, 2023-04-26 15:14 UTC

Citation for the original published paper (version of record):

Taghikhani, P., Buisman, K., Fager, C. (2020). Hybrid Beamforming Transmitter Modeling for Millimeter-Wave MIMO Applications. IEEE Transactions on Microwave Theory and Techniques, 68(11): 4740-4752. http://dx.doi.org/10.1109/TMTT.2020.2995657

N.B. When citing this work, cite the original published paper.

C2020 IEEE. Personal use of this material is permitted.

However, permission to reprint/republish this material for advertising or promotional purposes 


\title{
Hybrid Beamforming Transmitter Modeling for Millimeter-Wave MIMO Applications
}

\author{
Parastoo Taghikhani, Koen Buisman, Senior Member, IEEE, and Christian Fager, Senior Member, IEEE.
}

\begin{abstract}
Hybrid digital and analog beamforming is an emerging technique for high data rate communication at mm-wave frequencies. Experimental evaluation of such techniques is challenging, time-consuming and costly. This paper presents a hardwareoriented modeling method for predicting the performance of a mm-wave hybrid beamforming transmitter. The proposed method considers the effect of active circuit nonlinearity as well as the coupling and mismatch in the antenna array. It also provides a comprehensive prediction of radiation patterns and far-field signal distortions. Furthermore, it predicts the antenna input active impedance, considering the effect of active circuit loaddependent characteristics. The method is experimentally verified by a $29 \mathrm{GHz}$ beamforming subarray module comprising an analog beamforming Integrated Circuit (IC) and a $2 \times 2$ subarray microstrip patch antenna. The measurement results present good agreement with the predicted ones for a wide range of beam steering angles. As a use case of the model, far-field nonlinear distortions for different antenna array configurations are studied. The demonstration shows that the variation of nonlinear distortion versus steering angle depends significantly on the array configuration and beam direction. Moreover, the results illustrate the importance of considering the joint operation of beamforming ICs, antenna array, and linearization in the design of mm-wave beamforming transmitters.
\end{abstract}

Index Terms-Active antenna array, active impedance, beam steering, far-field nonlinear distortion, hybrid beamforming transmitter, millimeter wave.

\section{INTRODUCTION}

$\mathbf{T}$ HE large available bandwidth in millimeter wave (mmwave) bands can be explored to meet the demand for high data rate communication [1]-[3]. On the other hand, massive Multiple Input Multiple Output (MIMO) systems have been shown to be effective solutions for improving spectral efficiency and are now widely adopted in sub- $6 \mathrm{GHz}$ bands [4], [5]. Employing large scale antenna arrays along with the utilization of mm-wave bands enables high data rate communication [3], [6]. However, for a mm-wave massive MIMO system, implementing conventional digital beamforming architectures is not practical due to hardware complexity and high energy consumption. Hence, as a compromise, hybrid digital and analog beamforming architectures have been introduced [7]-[9]. In such configurations, each RF signal generation path is connected to multiple antenna elements via an analog

P. Taghikhani, K. Buisman, and C. Fager are with the Department of Microtechnology and Nanoscience, Chalmers University of Technology, SE-412 96 Gothenburg, Sweden (e-mails: \{parastoo;koen.buisman;christian.fager\}@ chalmers.se).

This work is a part of the Silicon-based Ka-band massive MIMO antenna systems for new telecommunication services (SILIKA) project, funded by the European Union's Horizon 2020 research and innovation program under the Marie Sklodowska Curie grant agreement No \#721732.



Fig. 1. Block diagram of a hybrid digital and analog beamforming transmitter.

beamforming unit. Therefore, the beamforming can be divided efficiently between the digital and analog domain to reduce the configuration complexity [10]. Fig. 1 shows a generic architecture of a hybrid beamforming transmitter consisting of baseband digital and RF analog beamformers. The analog beamforming unit includes power amplifiers (PAs) and phase shifters, which are normally integrated into an Integrated Circuit (IC). An option for evaluating hybrid beamforming transmitters' performance is to apply available circuit and EM simulation CAD tools [11]-[13]. CAD-based approaches combine the EM antenna model with a circuit simulator and allow performing circuit-level analysis of integrated structures [13]. However, using a CAD-based approach is a time-consuming and computationally expensive task for analysis of hybrid beamforming transmitters, particularly under modulated signal excitation. Each channel of a beamforming IC may include a large number of transistors interacting with adjacent channels in a large array configuration. For these situations, combining PA behavioral models with antenna characteristics is the key to a fast and accurate evaluation of transmitters [14].

A variety of PA behavioral models suitable for MIMO and phased array applications has been addressed in the literature [14]-[22]. However, only a limited number of studies have incorporated PA models into active antenna array simulations for evaluating transmitter performance [14], [18]-[22]. In [18], an iterative approach has been used to evaluate the active antenna array radiation pattern. The PA was approximated by the Poly-Harmonic Distortion (PHD) model [15] and used in the EM calculation of the antenna array radiation pattern. The simulation results showed that, for some specific 
beam steering angles, undesired sidelobes appeared due to the change in the delivered excitation of the array elements. However, experimental verification of the proposed technique is not reported. Moreover, incorporating PA behavior with numerical EM simulations adds complexity to the technique. Therefore, it is not an efficient method for subsequent design or optimization steps.

With the same focus on the radiation pattern, in [14] a methodology based on the combination of the antenna $S$ parameters and PHD models for the PAs was proposed. The method has been applied to a low frequency one-by-four linear antenna array to study its radiation patterns. However, the method was not employed to investigate signal distortion. Considering the importance of evaluating nonlinear signal distortion in active antenna arrays, a dual-input behavioral model with memory has been developed [19], and later used in [21], [22] to address transmitter signal nonlinearities. This prediction method has not been validated beyond $3 \mathrm{GHz}$. Furthermore, the radiation pattern has not been studied in conjunction with the signal distortion.

In [20], the behavior of an $8 \times 8$ phased array antenna has been predicted at $28 \mathrm{GHz}$ by using a memoryless scheme adapted from [19]. Simulation results showed that the distortion of the signal in the far-field changes with the steering angle. Although this study has been conducted for mm-wave applications, it does not include any experimental verification.

Various linearization approaches based on digital predistortion (DPD) have been experimentally studied for mmwave MIMO and hybrid beamforming transmitters [17], [23][30]. For beamforming transmitters, the results confirm that nonlinear distortion indeed depends on the steering angle. Therefore, several beam-oriented linearization schemes are proposed to compensate nonlinear distortion [24], [28], [29]. However, the results have not been evaluated against any theoretical prediction algorithm. This limits assessing the linearity and linearization techniques of a hybrid beamforming transmitter at the design stage before the hardware is built.

In this paper, first, a hardware-oriented methodology for prediction of a mm-wave hybrid beamforming transmitter performance is presented and experimentally validated. The behavior of the beamforming IC is characterized by loadpull measurements. The measured data are directly employed in an iterative algorithm to predict beamforming transmitter performance versus beam steering scenario. Thus, the beamforming IC representation is equally accurate for each load and independent of a mathematical expression. The radiation pattern and related far-field signal distortions are measured for the subarray module and validate the predicted results.

In the second part of the paper, the proposed analysis method is exploited in a simulation-based study to predict nonlinear distortion in various large-scale beamforming transmitter configurations. The study evaluates the linearity of the transmitters when used in combination with the corresponding ideal beam-dependent pre-distorters. This analysis resembles realistic cases where DPDs are applied to the beamforming transmitters. Thus, it also clarifies how the method can be used in the design stage to find a suitable array configuration from a linearity perspective.

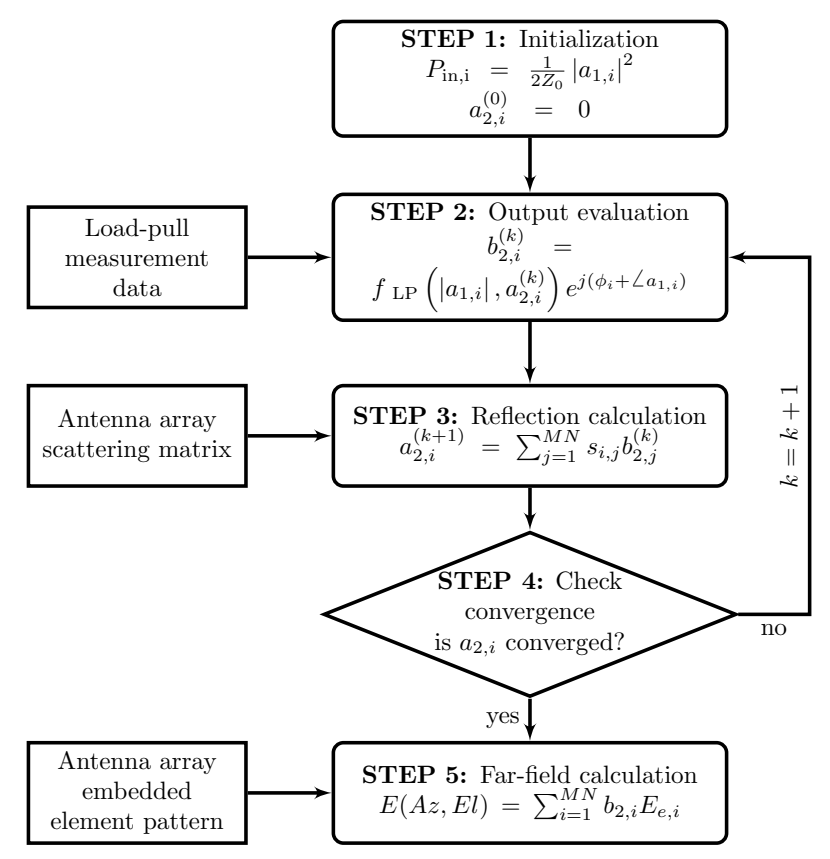

Fig. 2. Iterative algorithm for prediction of hybrid beamforming transmitter performance.

This paper is organized as follows. Section II presents an overview of the proposed analysis method for hybrid beamforming transmitters. This section also discusses the necessary characterization of the beamforming IC and subarray antenna. Section III includes measurement procedures and results for the beamforming IC and subarray antenna characterizations. Section IV discusses the process for the over the air (OTA) verification measurement and the obtained results. Based on the proposed analysis method, Section $\mathrm{V}$ presents a simulation study of large-scale beamforming transmitters and linearity performance. Finally, Section VI gives concluding remarks.

\section{HYBRID BEAMFORMING TRANSMITTER ANALYSIS}

The hybrid beamforming transmitter shown in Fig. 1 has $N$ RF chains, each of which consists of one beamforming unit and a $M$-element subarray antenna. In total there are $M \times N$ RF output channels connected to the large-scale antenna array. In this configuration, beamforming is performed in two stages. First digital precoding weights are applied to the RF input chains. Then, amplification and phase shifting is performed to the output channels via analog beamforming units.

In the $i$-th RF channel, $a_{1, i}$ is the input wave to the PA inside the beamforming unit and $a_{2, i}$ is the reflected wave at the output of the final stage PA. $a_{2, i}$ depends on both the mismatch between the PA and antenna and the mutual coupling between antenna elements. Two incident waves into the PA, $a_{1, i}$ and $a_{2, i}$, determine the output signal of the PA, $b_{2, i}$, which is also the incident wave to the $i$-th antenna element.

Implementing the interactions between the antenna array and analog beamforming units is critical for the correct prediction of the hybrid beamforming transmitter performance 
[31]. For this purpose, the beamforming units' behavior is here approximated with interpolation of load-pull measurement data, and an iterative algorithm is proposed to predict the overall performance. This section presents the algorithm and the necessary characterizations for implementing it.

\section{A. Beamforming Unit Characterization}

Load-pull data is used to characterize the RF channels of analog beamforming units. The load-pull measurements evaluate the changes in the output power, efficiency, and gain versus output load impedance. By also sweeping the input power for all specified loads, the nonlinearity of each RF channel is characterized. $b_{2}=f_{\mathrm{LP}}\left(\left|a_{1}\right|, a_{2}\right)$ is then created by linear interpolation of the measured data. This is later used to develop an iterative algorithm for prediction of the hybrid beamforming transmitter performance. Commonly, the $\mathrm{RF}$ channels of the beamforming units are similar. Therefore, it is typically sufficient to characterize one RF channel and use the extracted model for all other RF channels. If needed, non-identical channels can be represented with their specific function $b_{2, i}=f_{\mathrm{LP}, i}\left(\left|a_{1, i}\right|, a_{2, i}\right)$, where $i$ is the channel number.

\section{B. Antenna Array Characterization}

Multi-port $S$-parameters and embedded element radiation patterns of each antenna element are two key characteristics of the antenna array, which are needed to evaluate a hybrid beamforming transmitter. Both features can be extracted by either antenna measurements or full-wave EM simulation. Normally, to estimate the performance of an active array, simulated embedded element radiation patterns and $S$-parameters can be used both in the design and analysis stage.

\section{Iterative Algorithm}

Fig. 2 shows the flowchart of the iterative algorithm for the hybrid beamforming transmitter analysis. The analysis is performed in a $50 \Omega$ referenced power wave domain. For each specific level of the input power, $\left(P_{\mathrm{in}, i}=\frac{1}{2 Z_{0}}\left|a_{1, i}\right|^{2}, Z_{0}=\right.$ $50 \Omega$ ) the algorithm steps are as follows:

Step 1 (Initialization): First, the iteration is initialized under the assumption of no reflection and no coupling at the output of each RF channel, i. e. $a_{2, i}^{(0)}=0$. Based on the desired beamforming weights, proper phase-shifting for antenna elements, $\phi_{i}$, is specified.

Step 2 (Output evaluation): For the $k^{\text {th }}$ iteration we compute $b_{2, i}^{(k)}=f_{\mathrm{LP}}\left(\left|a_{1, i}\right|, a_{2, i}^{(k)}\right) e^{j\left(L_{1, i}+\phi_{i}\right)}, i=1: M \times N$, where $f_{\mathrm{LP}}(\cdot)$ is the load-pull based nonlinear behavioral model of the corresponding beamforming unit RF channel, see Section II-A.

Step 3 (Reflection calculation): After finding the output of the RF channels, $a_{2, i}^{(k+1)}$ should be modified considering the mismatch and the coupling between each RF channel using:

$$
a_{2, i}^{(k+1)}=\sum_{j=1}^{M N} s_{i, j} b_{2, j}^{(k)}
$$

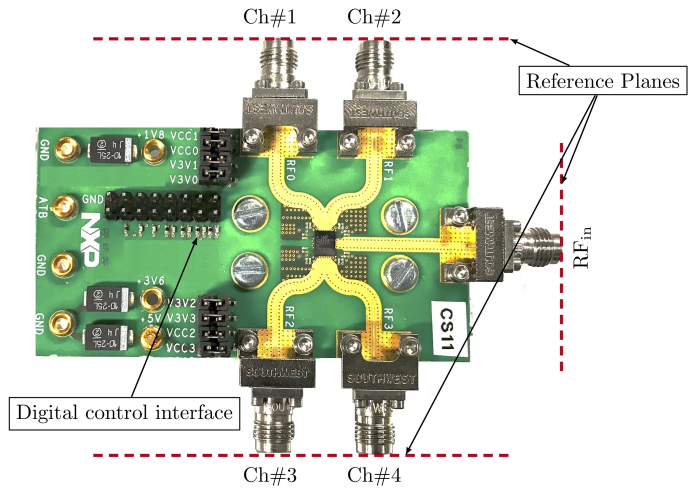

Fig. 3. Evaluation board of the quad-channel beamforming IC. $\mathrm{RF}_{\text {in }}$ is the input and $\mathrm{Ch \# 1}$ to $\mathrm{Ch \# 4}$ are four output RF channels. The digital control interface is used for phase and gain settings.

where $s_{i, j}$ are the elements of the antenna array $S$-parameters matrix.

Step 4 (Check convergence): In each iteration, $a_{2, i}$ depends on the output of other RF channels. The output of every RF channel depends on the represented load to its output. Steps 2 and 3 are therefore repeated for each RF channel until $a_{2, i}$ converges to a stationary level. The convergence rate depends on mismatch, mutual coupling level, and beamforming IC interpolation function. In each iteration, Step 3 has the main contribution to the calculation time. Thus, with the same number of iterations, the calculation time scales approximately linearly with the number of antennas.

Step 5 (Far-field calculation): The total radiated far-field can be predicted by superposition of the embedded element radiation patterns weighted by the $b_{2, i}$ excitations obtained [23]:

$$
E(A z, E l)=\sum_{i=1}^{M N} b_{2, i} E_{e, i}
$$

where $E_{e, i}$ is the embedded element pattern of the $i$-th antenna element $(i=1,2, \ldots, N)$ under unity excitation and when other elements are terminated in a matched load. The iterative algorithm effectively predicts the static performance of a hybrid beamforming transmitter including signal nonlinear distortion in the far-field and radiation pattern. If memory effects can be neglected, which could be a realistic approximation for relatively narrow band $\mathrm{mm}$-wave signals, the algorithm can also be used to predict the transmitter performance under modulated signal inputs. The iterative algorithm is then evaluating the performance at each base-band time-step of the modulated signal. In Section V, the analysis method is applied to linearize various transmitters and evaluate them with a modulated signal input.

Although analytical PA models proposed in [14], [19], [21] may be more time-efficient for investigating modulated signals, they do not perfectly emulate PA behavior for large mismatches. In large active antenna arrays, scan blindness due to large mismatches might occur for some steering angles [32]. Our proposed algorithm accurately predicts transmitter performance also when a large mismatch happens since the beamforming IC models do not rely on an analytical assumption. 


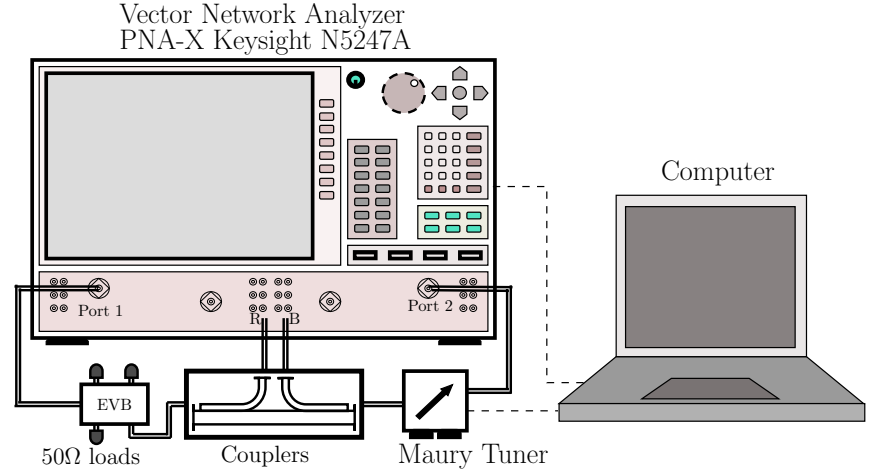

Fig. 4. Block diagram of the load-pull measurement setup.

PA models suitable for large mismatches have been proposed in [17]. However, the model has not been combined with the antenna array simulations.

\section{EVALUATING A MM-WAVE SUBARRAY MODULE PERFORMANCE}

In this section, a subarray module is developed and tested to validate the described analysis method in Section II. In this test case, the subarray module consists of a two-by-two planar microstrip patch antenna array, which is connected to an evaluation board (EVB) of a quad-channel analog beamforming IC from NXP Semiconductors, see Fig. 3. To assess the subarray module performance, first the beamforming IC and the subarray antenna are characterized as described in previous section. Then the proposed iterative algorithm predicts the subarray module performance for various beam steering scenarios. Next, over-the-air (OTA) measurements are performed to validate the predicted results in terms of the radiation pattern and far-field nonlinear distortion. In this work, the analysis has been limited to the fundamental frequency as the second harmonic contents are very small from the beamforming IC. It is, however, straightforward to extend the algorithm in Fig. 2 to include the effect of second harmonic signals, if needed.

\section{A. Beamforming IC}

The beamforming IC operates at 26.5-29.5 GHz. In the transmit mode, it consists of one input and four RF output channels, see Fig. 3. Each output channel has a digitally controlled buffer, amplifier and vector modulator (VM) for gain and phase control. With this possibility, independent analog beamforming weights can be applied to each of the output channels. The behavior of the beamforming IC channels, including the combined PA and VM, has been characterized using $S$-parameters and load-pull measurements. Thanks to the symmetrical design, all four RF channels have quite similar load-pull characteristics. The maximum variation between load-pull contours of different channels is about $0.15 \mathrm{~dB}$ in the output power and less than 1 degree in the output phase. $S$ parameters of all four RF channels have been measured under different gain and phase settings to evaluate their behavior in terms of gain and phase.

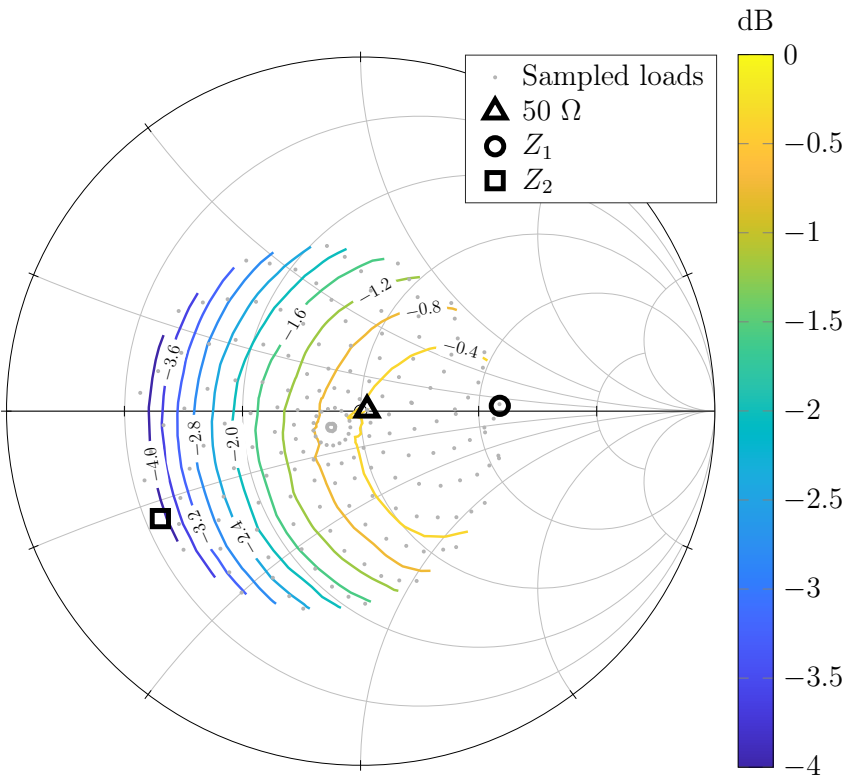

Fig. 5. Normalized delivered output power contours of the beamforming IC evaluation board which is achieved from load-pull measurement at $29 \mathrm{GHz}$. Markers are $Z_{1}=115+j 4 \Omega, Z_{2}=11-j 12 \Omega$ and $50 \Omega$. Gray points indicate loads used during load-pull characterization.

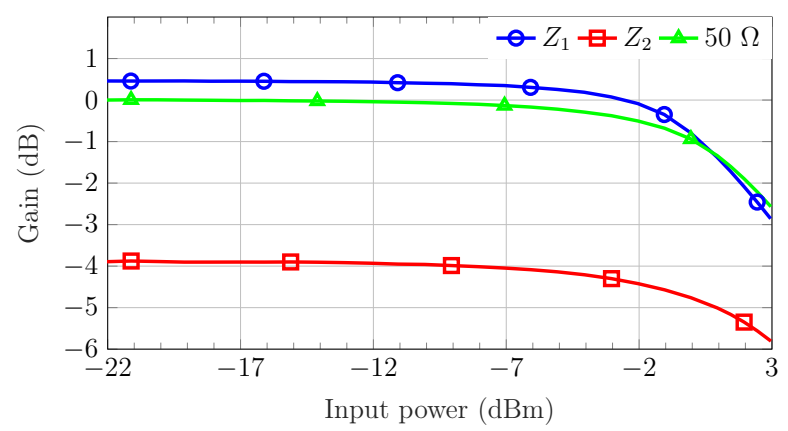

Fig. 6. Normalized AM/AM for different loads $Z_{1}=115+j 4 \Omega, Z_{2}=11-$ $j 12 \Omega$ and $50 \Omega$. The results are normalized to the small signal measurements with a $50 \Omega$ load.

1) Load-Pull Characterization Scenario: Fig. 4 shows the block diagram of the implemented load-pull measurement setup. A passive impedance tuner (Maury Microwave MT984A01) was used to tune the output load of the selected beamforming IC channel. The Vector Network Analyzer (VNA) is connected to a bi-directional coupler placed between the beamforming EVB and the tuner. This allows the load impedance presented to the EVB to be measured without the need for pre-characterization of the tuner. Power- and $S$ parameters calibration of this setup was done for the reference planes shown in Fig. 3. The Smith chart coverage area is constrained by the tuner, coupler- and cable losses. However, it will be shown later in Section IV-B that the sampled loads are enough to cover the impedances presented to the beamforming IC in the presence of the antenna element coupling, see Fig. 12 and Fig. 5. The channel under test has been set for maximum gain and zero phase during the measurements. All other channels were switched off and terminated in $50 \Omega$ 




Fig. 7. Beamforming IC calibration measurement. Solid red lines: Gain Setting (GS) are fixed values $31,41,51,63$ and phase is increased from 0 to 63. Dashed blue lines: Phase Setting (PS) for fixed values 2, 12, 22, 32, 52 with phase be increased from 0 to 63 .

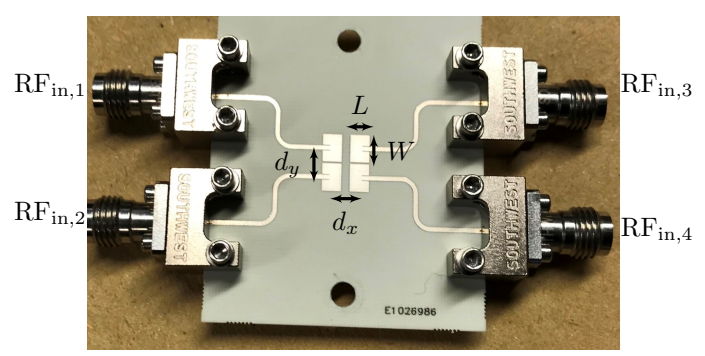

Fig. 8. Manufactured inset-fed microstrip two-by-two subarray antenna with element to element spacing, $d_{x}=d_{y}=0.4 \lambda_{0}=4 \mathrm{~mm}$. The antenna element dimensions are $w=4.2 \mathrm{~mm}$, and $l=4 \mathrm{~mm}$.

during the load-pull characterization. The input power to the beamforming IC is varied from $-22 \mathrm{dBm}$ to $3 \mathrm{dBm}$. The characterization measurement has done at $29 \mathrm{GHz}$.

For each of the tuner loads, $a_{1}, a_{2}$ and $b_{2}$ were measured versus input power. This forms the basis for the modeling of the beamforming IC behavior, as described in Section II-A i.e. $b_{2}=f_{\mathrm{LP}}\left(\left|a_{1}\right|, a_{2}\right)$. This function interpolates the measured data linearly. Extrapolation has not been applied since the interpolated data covers the needed range of loads.

Fig. 5 shows the normalized delivered output power contours obtained from the interpolated load-pull measurements data when the input power is $0 \mathrm{dBm}$. As it is shown, the delivered output power is $4 \mathrm{~dB}$ less than maximum for some of the loads tested. For a large range of loads around $50 \Omega$, the delivered output power is only $0.4 \mathrm{~dB}$ less than the maximum. The shape and center location of the load-pull contours depends on the IC technology, design criteria, operating frequency, etc.

Fig. 6 shows the measured AM/AM (amplitude output versus amplitude input) for three representative output load impedances, $Z_{1}=115+j 4 \Omega, Z_{2}=11-j 12 \Omega$ and $50 \Omega$. Similar behavior for the phase results has been observed, therefore, it is not presented here. As one can see, both the behavior and absolute level of AM/AM change significantly with the load impedance. The gain and phase compression vary between $-2 \mathrm{~dB}$ and $-3.2 \mathrm{~dB}$ and $8^{\circ}$ to $17^{\circ}$, respectively within the range of tested load impedances. This shows that the changes in the output load impedance can affect the

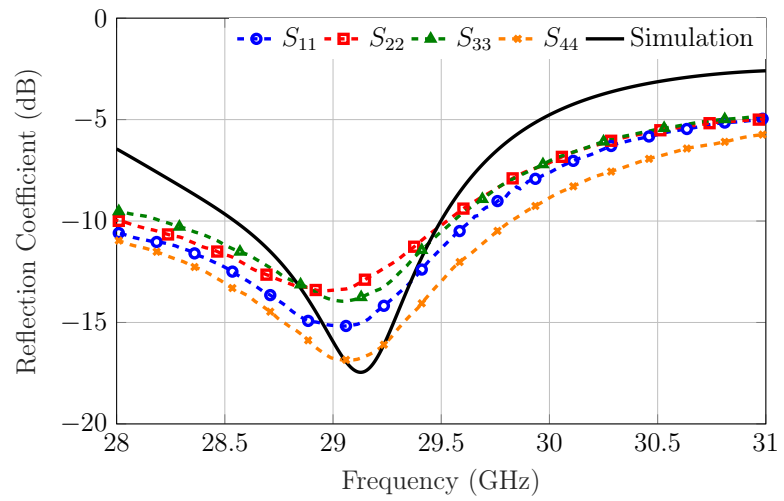

(a)



(b)

Fig. 9. Measurement and simulation of microstrip subarray antenna $S$ parameters: a) reflection coefficients, and b) coupling coefficients.

beamforming IC behavior significantly.

2) S-parameters Characterization and Linear Beamforming IC Calibration: S-parameters measurements of the beamforming IC show that the gains and phases of the output channels deviate from their set values. The deviations are different for each setting and each channel. Comprehensive $S$-parameters measurements of the beamforming IC in transmit mode have therefore been performed to establish the relationship between the gain and phase settings and the beamforming IC actual behavior.

Fig. 7 shows the measurement results of channel $1\left(\mathrm{RF}_{\text {out }, 1}\right)$ when, in one case, the gain is set to a fixed value and the phase is increased. In the other case, the phase is set to a fixed value and the gain is increased. The results show that the measured small signal phase deviates more than $17^{\circ}$ when the gain setting is incremented from 0 to 63 . Similarly, the measured small signal gain deviates about $1.5 \mathrm{~dB}$ (for the gain setting $=63$ ) when the phase setting is incremented from 0 to 63. Similar results are observed for the other channels.

Based on the presented $S$-parameters measurements, a calibration table was created to properly set beamforming IC gain and phase values for desired channel response. This was used in the OTA beam steering experiments. Although the calibration tables are slightly different for each channel, the characterization of channels load-pull is quite similar. 


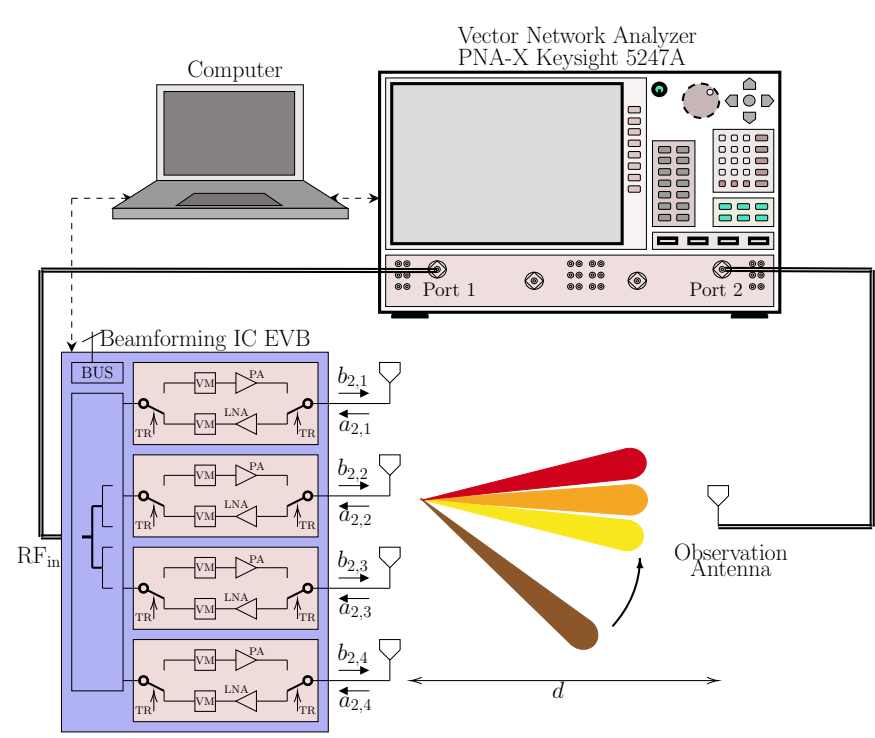

Fig. 10. The block diagram of the over-the-air measurement setup for hybrid beamforming analysis method validation. To the left, the subarray module, including antenna subarray and beamforming IC evaluation board, is located. To the right, an observation receiver antenna is connected to the VNA.

\section{B. Antenna array}

The microstrip patch antenna is compact, has wide beamwidth, and is therefore commonly selected as a radiating element in active antenna arrays. For the subarray module, a two-by-two microstrip rectangular patch array was designed and manufactured, see Fig. 8. The antenna element and array were designed in CST Microwave Studio for a resonant frequency around $29 \mathrm{GHz}$. A Rogers 4350 substrate with $0.25 \mathrm{~mm}$ thickness was used for manufacturing the subarray antenna.

As mentioned in Section II, both the $S$-parameters and embedded element radiation patterns of the subarray antenna are employed in the iterative algorithm. A four-port VNA (Keysight N5247 PNA-X) was used to measure the $S$-parameters of the manufactured antenna array, see Fig. 9. The highest measured mutual coupling is about $-12 \mathrm{~dB}$ for side-by-side elements. The coupling is below $-20 \mathrm{~dB}$ between elements facing each other and between the diagonal elements at $29 \mathrm{GHz}$. The measured reflection coefficients and mutual coupling are not symmetric although the antenna array consists of identical patches and there is a geometrical symmetry between all four elements. This is due to manufacturing and assembly tolerances. In general, there is a good agreement between measurement and simulation results. The resonant frequency is well predicted by the CST simulation and the highest measured coupling level is close to simulations around the resonant frequency. Embedded element patterns of the antenna elements were extracted from CST by post-processing the simulation data and used for the prediction of far-field distortion, as described in Section II.

\section{VERIFICATION MEASUREMENT RESULTS}

The subarray module performance was predicted using the iterative algorithm presented in Section II-C, and by applying
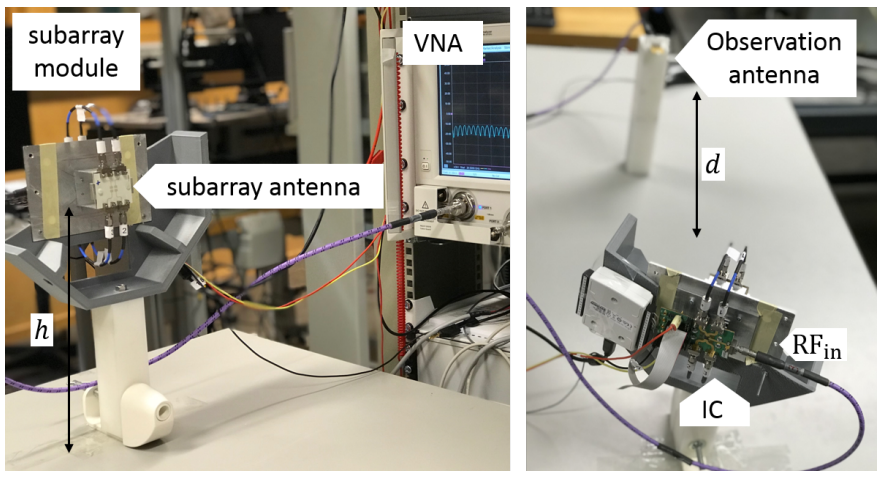

Fig. 11. The over-the-air measurement set up including subarray module, VNA and observation receiver antenna. $d$ is the spacing between subarray module as the transmitter, and receiver antenna. $h=30 \mathrm{~cm}$ is height of antennas above the measurement desk.

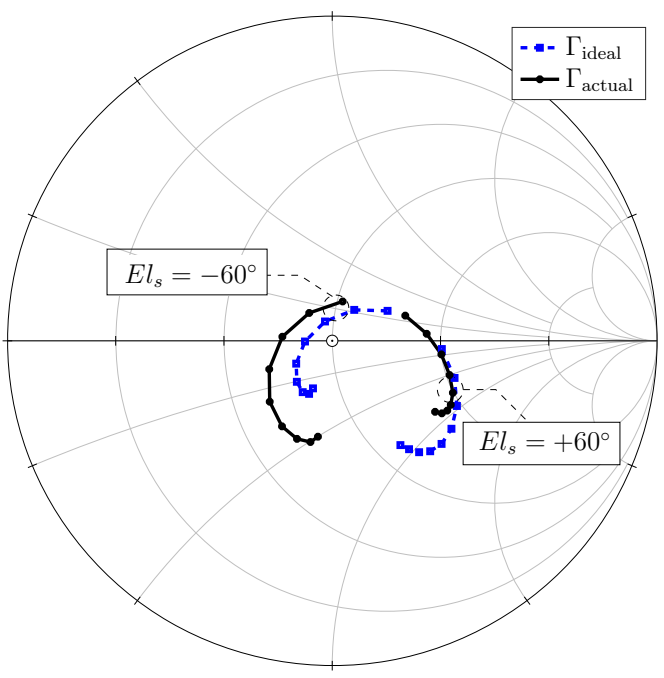

Fig. 12. Third antenna active impedance variation of antenna element obtained from hybrid beamforming analysis method ( $\Gamma_{\text {actual }}$, blue dashed line) and without considering joint PA and antenna interaction ( $\Gamma_{\text {ideal }}$, black line) for beam steering angle $E l=-60^{\circ}, 60^{\circ}-80^{\circ}<A z<0^{\circ}$.

the characterization results, which are reported above. OTA measurements were performed to experimentally validate the predicted far-field results.

\section{A. Experimental Setup}

Fig. 10 shows the block diagram of the OTA measurement setup. It includes the two-by-two subarray module as the transmitter, a single antenna as the receiver and a VNA for signal generation and reception. The input to the subarray module is a single tone signal at $29 \mathrm{GHz}$ generated by the VNA.

Fig. 11 shows a photo of the OTA measurement setup. The receiver observation antenna, which is connected to the VNA, is located in the far-field region of the transmitter. The far-field distance $d \approx \frac{2 D^{2}}{\lambda}=30 \mathrm{~cm}$, where $D=2 \mathrm{~cm}$ is the largest dimension of the subarray antenna.

The quad-channel beamforming IC can produce four waves with different phase and amplitude, corresponding to different analog beamforming weights, as described previously. In this 


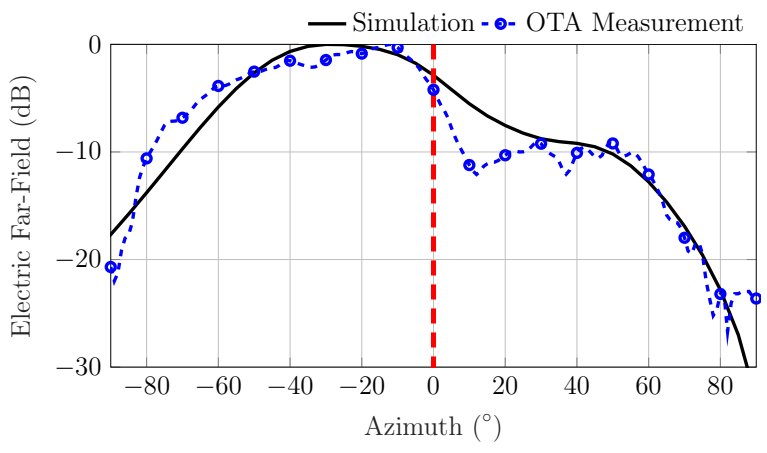

(a)

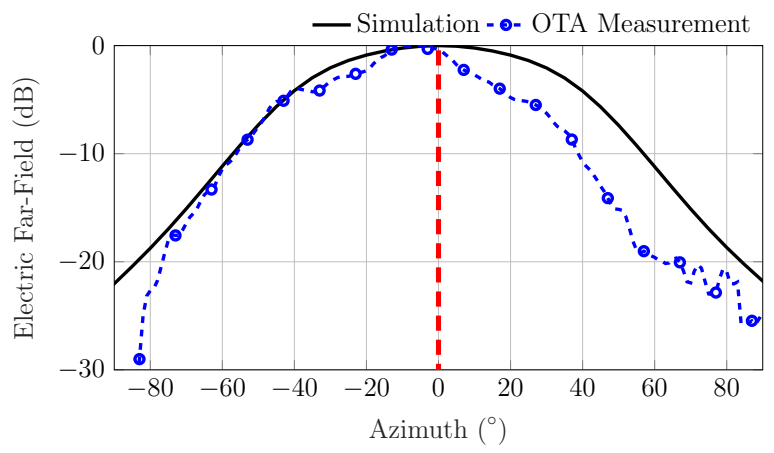

(b)

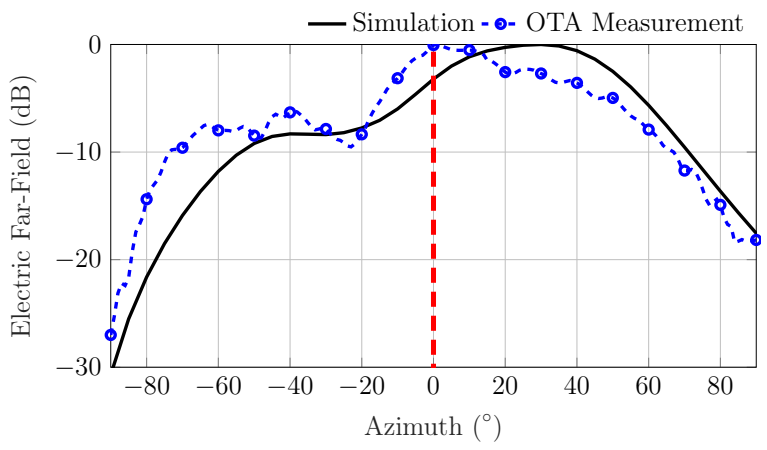

(c)

Fig. 13. H-plane radiation pattern $\left(E l=0^{\circ}\right)$ versus steering angle: a) $\left.\left(A z_{\mathrm{s}}, E l_{\mathrm{s}}\right)=\left(-30^{\circ}, 0^{\circ}\right), \mathrm{b}\right)\left(A z_{\mathrm{s}}, E l_{\mathrm{s}}\right)=\left(0^{\circ}, 0^{\circ}\right)$, and c) $\left(A z_{\mathrm{s}}, E l_{\mathrm{s}}\right)=$ $\left(30^{\circ}, 0^{\circ}\right)$. The red dashed line shows the boresight angle.

paper, we applied the phase shifting to steer the beam toward different azimuth and elevation angles. Proper phase values have been set to each channel using the $S$-parameters based calibration table, as described in Section III-A. The performance of the subarray module was measured at $29 \mathrm{GHz}$ and includes radiation pattern and AM/AM and AM/PM (output phase shift versus input amplitude) distortions in the far-field.

\section{B. Active Impedance}

The active impedance of an antenna array element is defined as the input impedance while all other elements are active. Normally, it is assumed that the source excitation performances are not affected by the output load [33]. Therefore, for a beam steering scenario with uniform amplitude excitations, the active reflection coefficient for the $i$-th antenna element



(a)

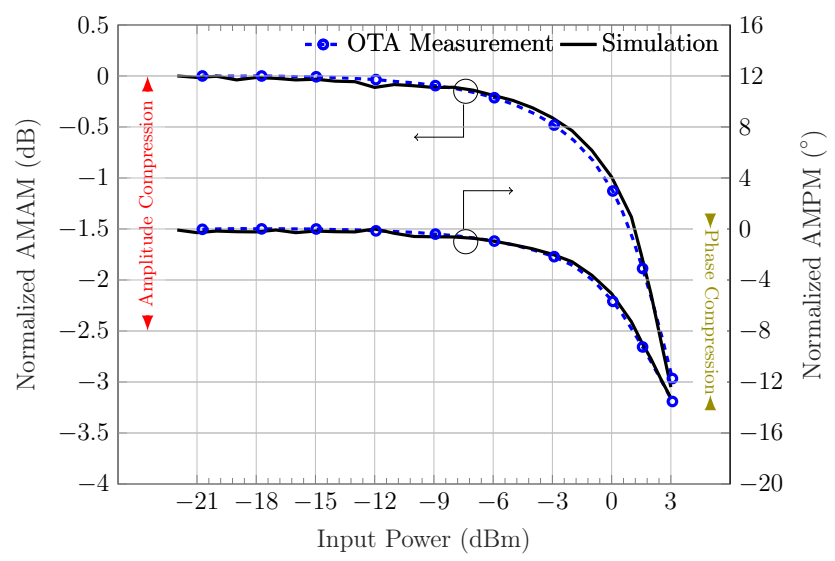

(b)

Fig. 14. $\mathrm{AM} / \mathrm{AM}$ and $\mathrm{AM} / \mathrm{PM}$ distortion in the far-field. OTA measurement vs simulation results for a) $\left(A z_{\mathrm{s}}, E l_{\mathrm{s}}\right)=\left(0^{\circ}, 0^{\circ}\right)$, and b) $\left(A z_{\mathrm{s}}, E l_{\mathrm{s}}\right)=$ $\left(-60^{\circ}, 0^{\circ}\right)$. Amplitude and phase compression are the difference between small signal and peak power gain and phase respectively.

$\left(\Gamma_{i, \text { ideal }}\right)$ is calculated as

$$
\Gamma_{i, \text { ideal }}=\frac{a_{2, i}}{b_{2, i}}=\frac{\sum_{j=1}^{N} s_{i, j} b_{2, j}}{b_{2, i}}=\sum_{j=1}^{N} s_{i, j} e^{j \phi_{j}}
$$

It can be seen that the active impedance for a specific steering angle depends on the antenna $S$-parameters, $s_{i, j}$, and applied phase shifting, $\phi_{j}$. However, source excitations are not ideal and their performance depends on the output load impedance, as is apparent from the load-pull contour plots in Fig. 5. Moreover, each output load has a different amplitude distortion characteristic as is shown in Fig. 6. The excitations delivered to the antenna elements, $b_{2, i}$, will therefore change from the ideal assumption and a joint solution involving the interactions between PAs and antenna array is needed. The active reflection coefficient expression in (3) should therefore be replaced by:

$$
\Gamma_{i, \text { actual }}=\frac{a_{2, i}}{b_{2, i}\left(a_{1, i}, a_{2, i}\right)}
$$

where $a_{2, i}$ and $b_{2, i}$ are obtained from the described method in Section II. Fig. 12 compares the active impedance from the hybrid beamforming analysis method calculation, $\Gamma_{\text {actual }}$, with $\Gamma_{\text {ideal }}$, for $E l_{\mathrm{s}}=-60^{\circ}, 60^{\circ}$ while $-80^{\circ}<A z_{\mathrm{s}}<0^{\circ}$. The 


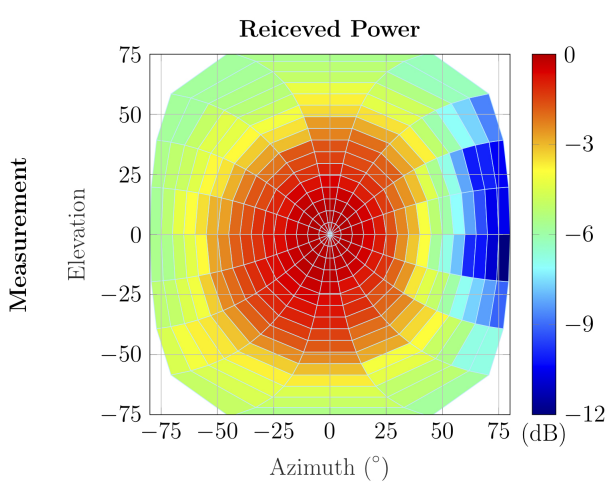

(a)

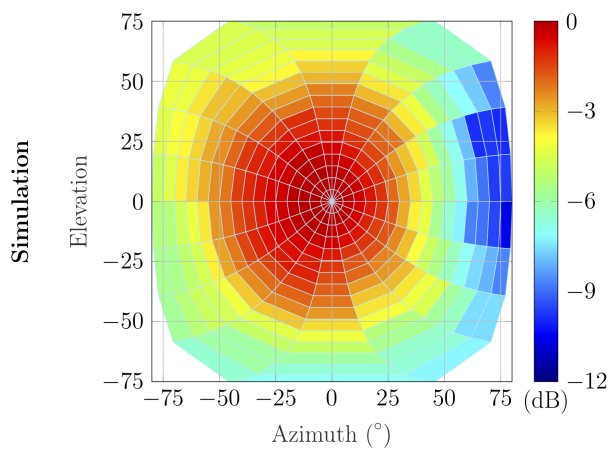

(b)

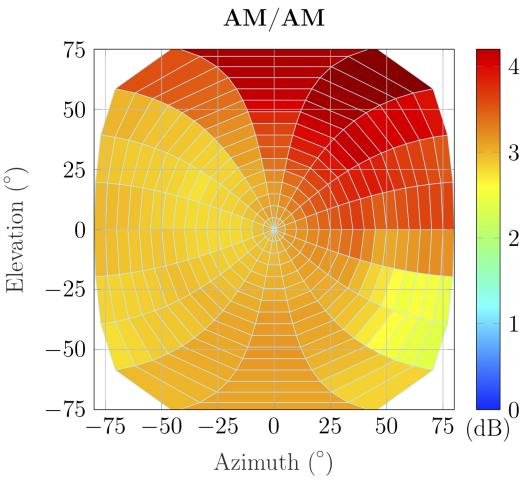

(c)

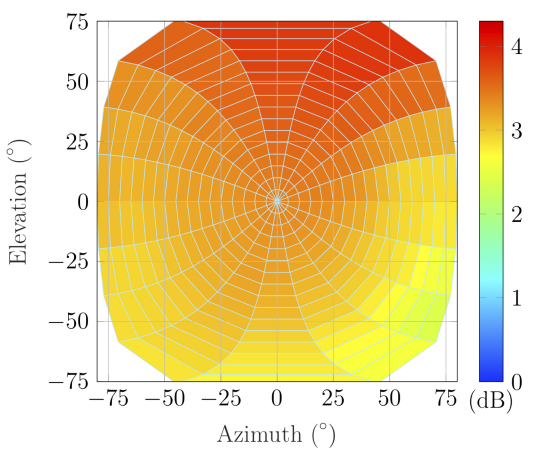

(d)

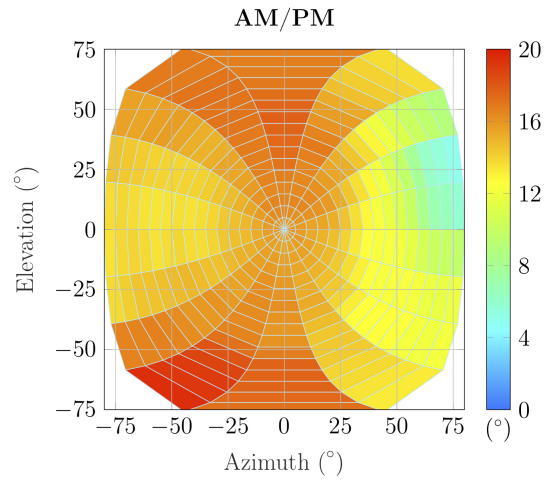

(e)

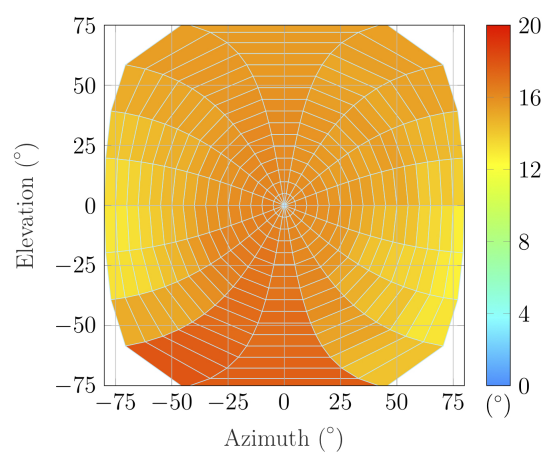

(f)

Fig. 15. Normalized received power together with amplitude and phase compression variation in the far-field for different azimuth and elevation steering angles, $\left|A z_{\mathrm{s}}\right|<80^{\circ},\left|E l_{\mathrm{s}}\right|<75^{\circ}$. Normalized received power: a) OTA experiment b) Simulation. Amplitude compression: c) OTA experiment d) Simulation. Phase compression: e) OTA experiment f) Simulation. The RMS error of amplitude and phase compression is $0.18 \mathrm{~dB}$ and 1.6 degrees, respectively.

$\Gamma_{\text {actual }}$ is not symmetrical for $E l_{\mathrm{s}}=-60^{\circ}, 60^{\circ}$ and shows considerable difference comparing to $\Gamma_{\text {ideal }}$ for the corresponding steering angles. This highlights the importance of considering nonlinear interactions between the antenna array and PAs in the calculation of active impedance.

\section{Far-Field Radiation Pattern}

Normally, antenna radiation pattern measurements should be performed in an anechoic chamber where EM absorbers effectively reduce any possible reflections. Due to the high propagation loss at mm-wave, reflections from the environment are relatively small and often negligible. In the OTA setup in Fig. 11 the transmitter and receiver antennas are placed $30 \mathrm{~cm}$ above the measurement desk plane and far from surrounding objects to minimize reflections. The receiver uses a microstrip Vivaldi antenna with a reflection coefficient below $-20 \mathrm{~dB}$ at $29 \mathrm{GHz}$. The transmitter antenna was rotated along its vertical axis. The relative values of the received power show the radiation pattern. Fig. 13 shows the $H$-plane radiation patterns obtained for steering angles $E l_{\mathrm{s}}=0^{\circ}$ and $A z_{\mathrm{s}}=-30^{\circ}, 0^{\circ}, 30^{\circ}$ with a fixed input power of $0 \mathrm{dBm}$. The prediction results were obtained from (2) using simulationbased embedded element patterns. There is a good agreement between the measurement and predicted results in terms of the direction of the steered beam and the shape of the radiation pattern. Some ripples in the measured radiation pattern are expected due to the non-ideal measurements setup and subarray module metal holders, which also add asymmetry to the shape of the radiation pattern. The environmental reflections create error comparable with conventional antenna measurements in an anechoic chamber. It should be noted that the predicted results are calculated using measured antenna $S$-parameters, simulated embedded element pattern and loadpull measurement data of beamforming IC. Inconsistency in any of this data will affect the agreement between predicted and measured results. The beam-width is quite large for the subarray module since the array consists of only two elements in the horizontal plane. Here, it is hard to see any considerable effect due to nonlinear distortion in the shape of the radiation pattern. However, a hybrid beamforming transmitter will use many subarray modules to create a highly directional antenna beam. Investigation of large scale arrays will be discussed in Section V.

\section{Far-Field Signal Nonlinear Distortion}

OTA measurements of AM/AM and AM/PM are used to evaluate how well the modeling approach predicts radiated signal distortion in the far-field for the subarray module. The subarray module was set to steer the beam to $\left|A z_{\mathrm{s}}\right|<80^{\circ}$ and $\left|E l_{\mathrm{s}}\right|<75^{\circ}$ angles. The observation receiver was not physically moved toward the transmitter steered the main beam due to practical difficulties. Furthermore, by fixing the position of the transmitter and receiver, measurement errors due to inaccurate positioning are minimized. Therefore, the 
observation receiver antenna measured radiated signal which is coming from the boresight angle for all steered beam cases, see red dashed lines shown in Fig 13.

Fig. 14 shows the normalized AM/AM and AM/PM farfield signal distortion for two specific steering angles i.e. $\left(A z_{\mathrm{s}}, E l_{\mathrm{s}}\right)=\left(0^{\circ}, 0^{\circ}\right)$ and $\left(-60^{\circ}, 0^{\circ}\right)$, predicted and measured with the OTA setup. The input power has been swept from -22 to $3 \mathrm{dBm}$. Here, the vertically polarized radiated electric field (co-polarization of the antenna array) is used to define the predicted far-field AM/AM and AM/PM using $\left(\frac{\left|E\left(0^{\circ}, 0^{\circ}\right)\right|}{\left|a_{1}\right|}\right)$ and $\left(\angle E\left(0^{\circ}, 0^{\circ}\right)-\angle a_{1}\right)$, respectively.

The OTA AM/AM and AM/PM distortion results correspond to the amplitude and phase of the measured VNA $S_{21}$ versus input power in the setup of Fig. 10. Fig. 14 shows that the predicted AM/AM and AM/PM results are in good agreement with the corresponding measurements. The calculated results using simulated instead of measured $S$-parameters show similar AM/AM behavior and about 2 degrees less AM/PM compression at the boresight direction. The difference between small signal and peak power gain and phase is denoted as "amplitude and phase compression" to quantify the AM/AM and AM/PM distortions behavior with two single values.

Fig. 15 shows the predicted and measured normalized received power as well as far-field amplitude and phase compression for beam steering angles $\left|A z_{\mathrm{s}}\right|<80^{\circ},\left|E l_{\mathrm{s}}\right|<75^{\circ}$. The received power for steering angles close to boresight are higher compared to $\left|A z_{\mathrm{s}}\right|>50^{\circ},\left|E l_{\mathrm{s}}\right|>50^{\circ}$. The measured and simulated result of the power level are in good agreement, see Fig. 15 (a) and (b).

The amplitude compression shows relatively higher values for most of the steering angles $\left|A z_{\mathrm{s}}\right|<80^{\circ}, E l_{\mathrm{s}}>20^{\circ}$ compared to the other steering angles which are similar to the predicted results, see Fig. 15 (c) and (d). Fig. 15 (e) shows that the phase compression is about 16 degrees for the steering angles $A z_{\mathrm{s}} \approx 0^{\circ},\left|E l_{\mathrm{s}}\right|<80^{\circ}$ and 13 degrees for $\left|A z_{\mathrm{s}}\right|<80^{\circ}, E l_{\mathrm{s}} \approx 0^{\circ}$, which agrees well with the simulation results. For extreme steering angles, it is expected that the difference between simulated and measured embedded element radiation patterns increases. The main reason for this can be the environmental effects and hardware impairments that affect the measured radiation pattern at lower levels. Fig. 15 (a) and (b) shows that the received signal level is $12 \mathrm{~dB}$ less for $60^{\circ}<A z_{\mathrm{s}}<80^{\circ},\left|E l_{\mathrm{s}}\right|<50^{\circ}$ steering angle compared to boresight angle. This may explain the larger discrepancy between measurement and simulation for the corresponding steering directions in Fig. 15 (e) and (f). The comparison between measured and simulated results shows that the RMS error of the amplitude and phase compression are $0.18 \mathrm{~dB}$ and 1.6 degrees, respectively. Therefore, in general, the difference between the predicted results and measured one is acceptable considering uncertainties in positioning and loadpull measurements, together with other hardware impairments associated with mm-wave measurements.

In this section, the analysis method for the hybrid beamforming transmitter is validated for a subarray module. The results confirm the analysis validity for a wide range of steering angles. For this test case, the analysis algorithm converged within 10 iterations. The MATLAB calculation time is about 0.5 second for each steering angle with a conventional desktop computer. The measurements have not been performed in the main steered beams due to practical challenges. However, the proposed measurement scenario suffices to validate the analysis method.

The presented method can be directly used to predict radiation patterns and static nonlinear signal distortion in the far-field. Moreover, it can also be used to investigate the dynamic nonlinear behavior of the modulated signal if the signal bandwidth is relatively low. An experimental study with modulated signals was not performed due to the limited availability of suitable instruments. Still, the single tone continuous wave $(\mathrm{CW})$ measurements effectively validate the proposed modeling method. In the following section, we will show how the method can be applied in the early stages of a beamforming transmitter design.

\section{BEAMFORMING TRANSMITTER LINEARITY STUDY}

In this section, with the help of the proposed method in Section II, different large-scale hybrid beamforming transmitters are evaluated from a linearity perspective. This study demonstrates the potential of our proposed method in a typical design application. Here, the impact of different array configurations on far-field nonlinear distortion is investigated when the transmitter is linearized.

\section{A. Beamforming Transmitter Configurations}

Fig. 16 illustrates the $\mathrm{N}_{\mathrm{x}} \times \mathrm{N}_{\mathrm{y}}$ element array configuration which is considered as the beamforming transmitter. $\mathrm{N}_{\mathrm{x}}$ and $\mathrm{N}_{\mathrm{y}}$ are the number of elements in $\mathrm{x}$ and $\mathrm{y}$ directions, respectively. The transmitters building blocks are $2 \times 2$ subarray modules, which are driven by individual beamforming ICs. In a practical hardware architecture, beamforming ICs will be placed at the back side of the antenna array, see Fig. 16.

Two antenna array configurations, $8 \times 8$ and $16 \times 4$ are designed in CST and studied here. For both configurations, a Rogers Duroid 5880 substrate with $0.25 \mathrm{~mm}$ thickness is used. The array elements are pin-fed patch antennas with below $-25 \mathrm{~dB}$ return loss at the center frequency of $29 \mathrm{GHz}$. The simulated coupling levels between adjacent elements in $\mathrm{x}$ and y direction are about $-12 \mathrm{~dB}$ and $-20 \mathrm{~dB}$, respectively. The linearity of the transmitters is being evaluated for a beam steering scenario using the proposed algorithm in Section II. The inputs to the algorithm, such as the antenna array $S$ parameters and embedded element patterns, are extracted from EM simulations. The beamforming IC is the one that was characterized in Section III-A1.

\section{B. Transmitter Linearization}

The importance of mitigating linearity in MIMO transmitters has lead to the development of various of DPD approaches [17], [23]-[30]. For hybrid beamforming transmitters, beamdependent DPD schemes have shown to be an efficient solution [24], [28], [29]. Therefore, a similar beam-dependent DPD has 


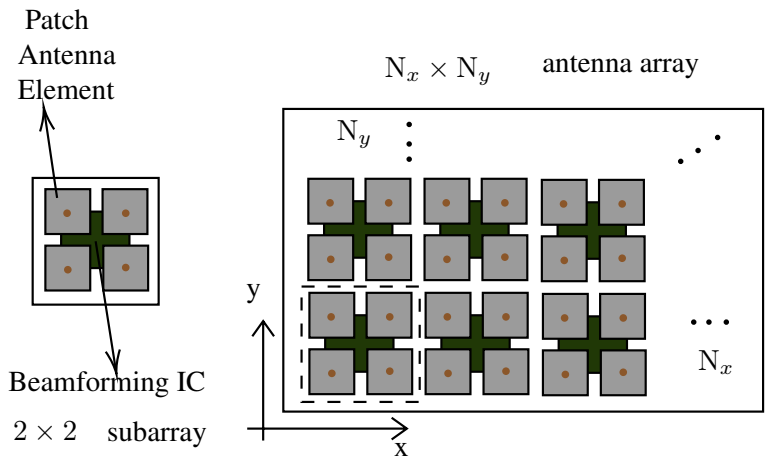

Fig. 16. $\mathrm{N}_{\mathrm{x}} \times \mathrm{N}_{\mathrm{y}}$ array considered as hybrid beamforming transmitters. Each $2 \times 2$ subarray module is fed with a quad channel beamforming IC.

been assumed in this study when we evaluate the transmitter linearity, i.e. an ideal linearizer is designed based on predicted far-field nonlinear distortion, and then the behavior of linearized transmitter is evaluated for a beam-steering scenario. This specific study demonstrates the potential of the proposed method of Section. II for analysis of design tradeoffs.

Fig. 17 shows the structure of the considered beamforming transmitter utilizing an ideal pre-distorter. The ideal predistorter is designed to linearize the radiated signal in one specific beam direction $\left(A z_{\mathfrak{t}}, E l_{\mathfrak{t}}\right)$. The following steps are performed to create the ideal pre-distorter function and to apply it to beamforming transmitters.

- Step 1 (Transmitter evaluation) First, the beamforming transmitter radiated far-field is evaluated for one specific steered beam. $\left(A z_{\mathrm{t}}, E l_{\mathrm{t}}\right)$ represents the direction of the target beam or user direction to be fully linearized. $E\left(A z_{\mathrm{t}}, E l_{\mathrm{t}},\left|a_{1}\right|\right)$ is the main beam electric field value, which is obtained by applying the method described in Section II. The transmitter input is a single tone signal with a power of $P_{\text {in }}=\frac{1}{2 Z_{0}}\left|a_{1}\right|^{2}$. $P_{\text {in }}$ is swept until the transmitter is a few $\mathrm{dBs}$ in compression.

- Step 2 (Obtain ideal pre-distorter) The goal is to linearize the target steered beam at $\left(A z_{\mathrm{t}}, E l_{\mathrm{t}}\right)$. Thus, the ideal pre-distorter function is obtained by swapping input and output of the $E\left(A z_{\mathfrak{t}}, E l_{\mathfrak{t}},\left|a_{1}\right|\right)$ function. $\left|E\left(A z_{\mathfrak{t}}, E l_{\mathfrak{t}},\left|a_{1}\right|\right)\right|$ as the input, and $\left|a_{1}\right|$ as the output are interpolated to obtain predistorter AM/AM function. The pre-distorter AM/PM function is the minus of $\angle E\left(A z_{\mathrm{t}}, E l_{\mathrm{t}},\left|a_{1}\right|\right)$. See Fig. 17 (b) and (c) for the conceptual representation of the pre-distorter AM/AM and $\mathrm{AM} / \mathrm{PM}$ functions.

- Step 3 (Linearity evaluation) The behavior of the beamforming transmitter combined with the ideal pre-distorter is evaluated for all beam steering cases. $\hat{E}_{\mathrm{s}}(n)$ is the desired linear far-field signal at the time instance $n . E_{\mathrm{s}}(n)$ is the main beam value of an individual steered beam. The normalized mean square error (NMSE) is considered as the figure of merit for evaluating the linearity of each steering beam. The NMSE is calculated by:

$$
\mathrm{NMSE}=\frac{\sum_{n=1}^{N}\left|\alpha E_{\mathrm{s}}(n)-\hat{E}_{\mathrm{s}}(n)\right|^{2}}{\sum_{n=1}^{N}\left|\alpha E_{\mathrm{s}}(n)\right|^{2}}
$$

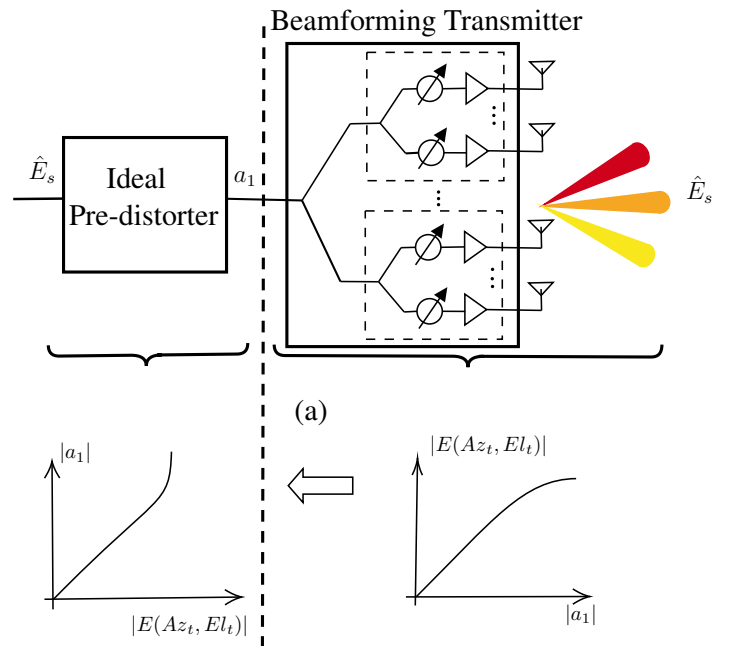

(b)
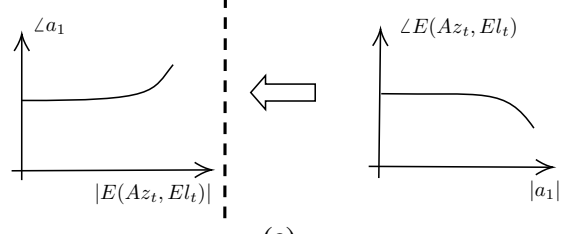

(c)

Fig. 17. a) Structure of hybrid beamforming transmitter utilizing an ideal predistorter. b) The conceptual representation of the transmitter transfer AM/AM function and pre-distorter corresponding one c) AM/PM representation.

where $N$ is the total number of time samples. $\alpha$ is a complex scaling factor which is calculated for each steered beam to eliminate the effects of gain and output power variations in different steering directions.

\section{Simulation Results}

A single carrier Long Term Evolution (LTE) communication signal with $8.5 \mathrm{~dB}$ Peak-to-Average Power Ratio (PAPR) and $20 \mathrm{MHz}$ bandwidth is selected as the desired far-field signal of the structure in Fig. 17 (a). The signal bandwidth is relatively narrow. Therefore, the single frequency characterization of the antenna array and beamforming IC is sufficient for evaluating the pre-distorted beamforming transmitter. The evaluation algorithm converges within 10 iterations for the $8 \times 8$ and $16 \times 4$ antenna array. Each iteration takes about 11 times longer compared to the studied $2 \times 2$ array in Section III. This confirms that the number of iterations stays the same and independent of the array size and, the total simulation time scales approximately linear with the number of antennas.

The ideal pre-distorter is designed based on the procedure described in Section V-A. The $16 \times 4$ transmitter is linearized for the steering angles $\left(A z_{\mathrm{t}}, E l_{\mathrm{t}}\right)=(0,0)^{\circ},(50,0)^{\circ},(30,25)^{\circ}$ and the $8 \times 8$ transmitter is linearized for $\left(A z_{\mathrm{t}}, E l_{\mathrm{t}}\right)=(0,0)^{\circ}$. Fig. 18 (a) shows the linearity (NMSE) of the $8 \times 8$ linearized beamforming transmitter at $\left(A z_{\mathrm{t}}, E l_{\mathrm{t}}\right)=(0,0)^{\circ}$ direction for steered beams $\left|A z_{\mathrm{s}}\right|<80^{\circ},\left|E l_{\mathrm{s}}\right|<75^{\circ}$. Per design, in the direction $\left(A z_{\mathrm{t}}, E l_{\mathrm{t}}\right)=(0,0)^{\circ}$, the transmitter with DPD is ideally linear. The linearity performance degrades for other beam directions. The NMSE variation between two beams at $\left(A z_{\mathrm{s}}, E l_{\mathrm{s}}\right)=(20,0)^{\circ}$ and $(70,20)^{\circ}$ is about $30 \mathrm{~dB}$. It is 


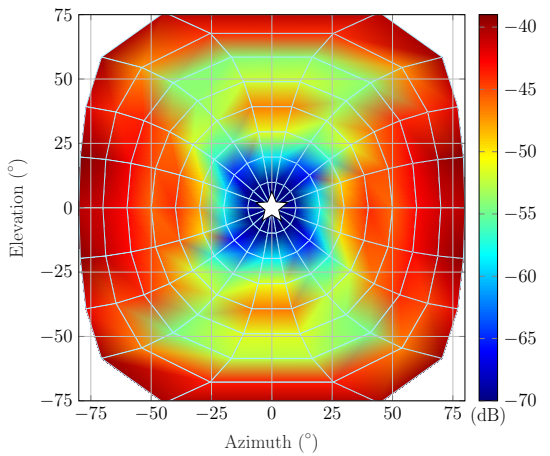

(a)

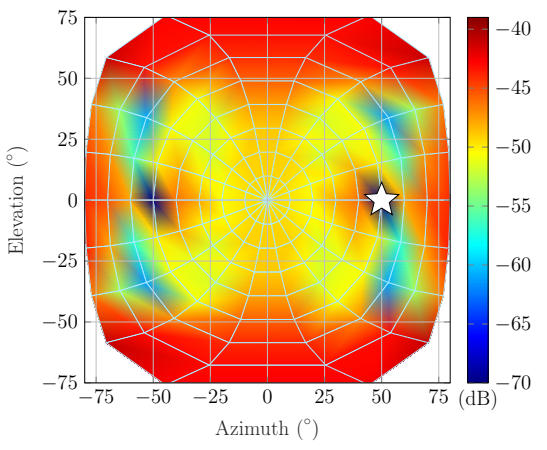

(c)

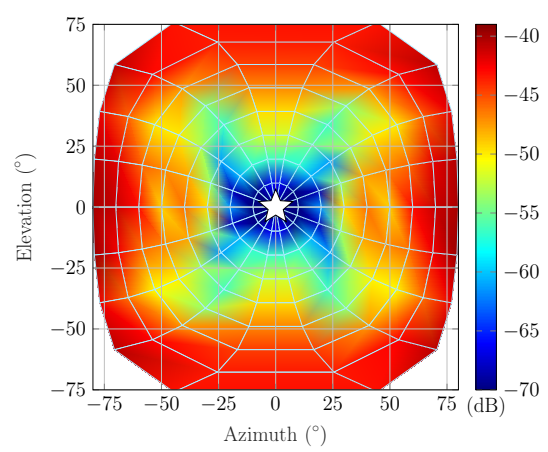

(b)

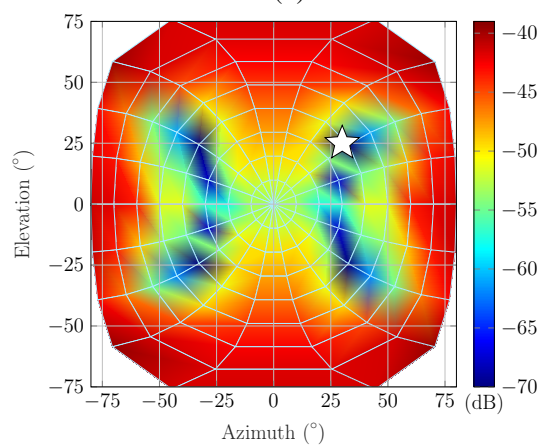

(d)

Fig. 18. Nonlinear distortion in the far-field versus steering angle for a) $8 \times 8$ array linearized for the beam at $\left.\left(A z_{\mathrm{t}}, E l_{\mathrm{t}}\right)=(0,0)^{\circ}, \mathrm{b}\right) 16 \times 4$ array linearized for $\left(A z_{\mathrm{t}}, E l_{\mathrm{t}}\right)=(0,0)^{\circ}$, c) $16 \times 4$ array linearized for $\left(A z_{\mathrm{t}}, E l_{\mathrm{t}}\right)=(50,0)^{\circ}$, and d) $16 \times 4$ array linearized for $\left(A z_{\mathrm{t}}, E l_{\mathrm{t}}\right)=(30,25)^{\circ}$. The results are presented in terms of normalized mean square error for a LTE signal with 8.5 peak-to-average power ratio. The white filled star represents the direction that the beam-dependent DPD is optimized for.

interesting to observe that the linearity degrades more rapidly for the beams steered toward large azimuth angles compared to large elevation angles.

Fig. 18 (b) presents the results for a $16 \times 4$ array configuration, which is linearized at the boresight direction. Compared to $8 \times 8$ array behavior, the NMSE for large elevation angles, i.e. $\left|E l_{\mathrm{s}}\right|>40^{\circ}$, is higher. Generally, it is clear that the array configuration and coupling level between elements in $\mathrm{x}$ or $\mathrm{y}$ direction affect the linearity variation.

In another case-study, the ideal pre-distorter is designed to linearize the $16 \times 4$ array for the beams in $\left(A z_{\mathrm{s}}, E l_{\mathrm{s}}\right)=(50,0)^{\circ}$ and $(30,25)^{\circ}$ directions, see Fig. 18 (c) and (d) respectively. In general, Fig. 18 illustrates that linearity behavior has a diagonal symmetry. This happens because beams at $\left(A z_{\mathrm{s}}, E l_{\mathrm{s}}\right)$ and $\left(-A z_{\mathrm{s}},-E l_{\mathrm{s}}\right)$ are identical for an equally spaced ideal rectangular array. The antenna array $S$-parameters is ideally symmetric and matching is identical for all elements. Furthermore, the beamforming IC channels are assumed to be identical. In practice, the antenna element and beamforming IC channels are not identical which could be accounted for in the model. The comparison between the results in Fig. 18 (b), (c) and (d) shows that for a fixed array configuration, the linearity behavior depends significantly on the DPD optimized beam direction. The results confirm the importance of such studies for a beamforming transmitter in the design stage.

In a multi-user hybrid beamforming transmitter, antenna array sub-sections send individual RF signals to users. For such scenarios, the experimental results in [25] confirm that each user can be linearized independently and beams typically do not interfere with each other. Therefore, the investigation in this section can be generalized also to a multi-user beamforming case. Certainly, the results here depends strongly on the beamforming IC and antenna array characteristics. However, such a study can be applied to any hybrid beamforming transmitter to determine a suitable antenna array configuration or linearization approach. In fact, the variation of beam linearity with steering angle shows how the PA and antenna array interaction influences the large active antenna array performance. Overall, such type of studies can be used to determine an optimum design for a hybrid beamforming transmitter.

\section{CONCLUSIONS}

In this paper, we combined a load-pull based nonlinear model of an analog beamforming unit with antenna characteristics to develop an iterative algorithm for prediction of hybrid beamforming transmitter performance at mm-wave frequencies. The proposed technique is based on the interpolation of load-pull measured data and is shown to be a feasible method for modeling at mm-wave frequencies. The analysis method predicts far-field radiation patterns and static nonlinear distortion of the hybrid beamforming transmitters. It accurately calculates the active impedance by considering the effect of antenna and PA interactions.

A $29 \mathrm{GHz}$ subarray module including a $2 \times 2$ microstrip patch antenna array and a beamforming IC was developed to validate the hybrid beamforming analysis method. The OTA 
measurement results prove the validity of the method as both radiation pattern and far-field distortion are in good agreement with simulation results. This demonstrates the strength of the method in a larger perspective compared to similar works. The active impedance of the proposed subarray module is extracted by the proposed approach and shows a significant difference compared to regular calculation methods.

Finally, the nonlinear distortion of the designed $29 \mathrm{GHz}$ large-scale beamforming transmitters with various configurations has been predicted in a realistic beam-linearized application scenario. The study reveals that array dimensions, as well as the linearization, affect the nonlinear distortion level and its variation versus steering angle. Therefore, these effects have to be accounted for when designing a beamforming transmitter.

The proposed analysis method can provide design guides for developing large-scale arrays and can be employed for highlevel optimization with low computational effort. Furthermore, the developed method can effectively be used for investigating any hybrid digital and analog beamforming scenarios by applying digital and analog beamforming weights to the user data.

\section{ACKNOWLEDGMENT}

The authors wish to thank NXP Semiconductors for providing the quad-channel beamforming IC evaluation board.

\section{REFERENCES}

[1] S. K. Yong and C.-C. Chong, "An overview of multigigabit wireless through millimeter wave technology: Potentials and technical challenges," EURASIP J. Wireless Commun. Network., vol. 2007, no. 1, pp. 50-50, 2007.

[2] S. Rangan, T. S. Rappaport, and E. Erkip, "Millimeter-wave cellular wireless networks: Potentials and challenges," Proceedings of the IEEE, vol. 102, no. 3, pp. 366-385, 2014.

[3] T. S. Rappaport et al., "Millimeter wave mobile communications for $5 \mathrm{G}$ cellular: It will work!" IEEE Access, vol. 1, no. 1, pp. 335-349, 2013.

[4] E. G. Larsson, O. Edfors, F. Tufvesson, and T. L. Marzetta, "Massive mimo for next generation wireless systems," IEEE Commun. Mag., vol. 52, no. 2, pp. 186-195, 2014.

[5] F. Rusek et al., "Scaling up MIMO: Opportunities and challenges with very large arrays," IEEE Signal Process. Mag., vol. 30, no. 1, pp. 40-60, 2013.

[6] A. Gupta and R. K. Jha, "A survey of 5G network: Architecture and emerging technologies," IEEE Access, vol. 3, pp. 1206-1232, 2015.

[7] W. Roh et al., "Millimeter-wave beamforming as an enabling technology for $5 \mathrm{G}$ cellular communications: Theoretical feasibility and prototype results," IEEE Commun. Mag., vol. 52, no. 2, pp. 106-113, 2014.

[8] A. Alkhateeb, J. Mo, N. Gonzalez-Prelcic, and R. W. Heath, "MIMO precoding and combining solutions for millimeter-wave systems," IEEE Commun. Mag., vol. 52, no. 12, pp. 122-131, 2014.

[9] F. Sohrabi and W. Yu, "Hybrid analog and digital beamforming for mmWave OFDM large-scale antenna arrays," IEEE J. Select. Areas Commun., vol. 35, no. 7, pp. 1432-1443, 2017.

[10] R. W. Heath, N. Gonzalez-Prelcic, S. Rangan, W. Roh, and A. M Sayeed, "An overview of signal processing techniques for millimeter wave MIMO systems," IEEE J. Seleced Topic Signal Process., vol. 10, no. 3, pp. 436-453, 2016.

[11] V. Rizzoli, A. Costanzo, P. Spadoni, F. Donzelli, D. Masotti, and E. M. Vitucci, "A CAD procedure for MIMO link estimation by the combination of nonlinear, electromagnetic and propagation analysis techniques," in Proc. IEEE MTT-S Int. Microw. Symp. Dig., 2008, pp. 927-930.

[12] V. Rizzoli, A. Costanzo, D. Masotti, M. Aldrigo, F. Donzelli, and V. Degli Esposti, "Integration of non-linear, radiation, and propagation CAD techniques for MIMO link design," J. Int. Microw. Wireless Tech., vol. 4, no. 2, pp. 223-232, 2012.
[13] H. Aliakbari, A. Abdipour, A. Costanzo, D. Masotti, R. Mirzavand, and P. Mousavi, "Far-field-based nonlinear optimization of millimeter-wave active antenna for 5G services," IEEE Trans. Microw. Theory Techn., vol. 67, no. 7, pp. 2985-2997, 2019.

[14] G. Z. El Nashef, F. Torres, S. Mons, T. Reveyrand, T. Monédière, E. Ngoya, and R. Quéré, "EM/circuit mixed simulation technique for an active antenna," IEEE Antennas Wireless Propag. Lett., vol. 10, pp. 354-357, 2011

[15] D. Root et al., "Polyharmonic distortion modeling," IEEE Microw. Mag. vol. 7, no. 3, pp. 44-57, 2006.

[16] S. Amin, P. N. Landin, P. Händel, and D. Rönnow, "Behavioral modeling and linearization of crosstalk and memory effects in RF MIMO transmitters," IEEE Trans. Microw. Theory Techn., vol. 62, no. 4, pp. 810-823, 2014.

[17] S. K. Dhar, A. Abdelhafiz, M. Aziz, M. Helaoui, and F. M. Ghannouchi, "A reflection-aware unified modeling and linearization approach for power amplifier under mismatch and mutual coupling," IEEE Trans. Microw. Theory Techn., no. 99, pp. 1-11, 2018.

[18] M. Romier, A. Barka, H. Aubert, J.-P. Martinaud, and M. Soiron, "Load-pull effect on radiation characteristics of active antennas," IEEE Antennas Wireless Propag. Lett., vol. 7, pp. 550-552, 2008.

[19] C. Fager, X. Bland, K. Hausmair, J. C. Cahuana, and T. Eriksson, "Prediction of smart antenna transmitter characteristics using a new behavioral modeling approach," in Proc. IEEE MTT-S Int. Microw. Symp. (IMS), 2014, pp. 1-4.

[20] C. Fager, K. Hausmair, K. Buisman, K. Andersson, E. Sienkiewicz, and D. Gustafsson, "Analysis of nonlinear distortion in phased array transmitters," in Proc. Workshop Integ. Nonlinear Microw. Millim. Wave Circuits (INMMiC), Apr. 2017, pp. 1-4.

[21] K. Hausmair, S. Gustafsson, C. Sánchez-Pérez, P. N. Landin, U. Gustavsson, T. Eriksson, and C. Fager, "Prediction of nonlinear distortion in wideband active antenna arrays," IEEE Trans. Microw. Theory Techn. vol. 65, no. 11, pp. 4550-4563, 2017.

[22] F. M. Barradas, P. M. Tomé, J. M. Gomes, T. R. Cunha, P. M. Cabral, and J. C. Pedro, "Power, linearity, and efficiency prediction for MIMO arrays with antenna coupling," IEEE Trans. Microw. Theory Techn., vol. 65, no. 12 , pp. 5284-5297, 2017.

[23] K. Hausmair, P. N. Landin, U. Gustavsson, C. Fager, and T. Eriksson, "Digital predistortion for multi-antenna transmitters affected by antenna crosstalk," IEEE Trans. Microw. Theory Techn., vol. 66, no. 3, pp. 1524 $1535,2018$.

[24] C. Yu et al., "Full-angle digital predistortion of 5G millimeter-wave massive MIMO transmitters," IEEE Trans. Microw. Theory Techn., vol. 67, no. 7, pp. 2847-2860, 2019.

[25] E. Ng, A. B. Ayed, P. Mitran, and S. Boumaiza, "Single-input singleoutput digital predistortion of multi-user RF beamforming arrays," in IEEE MTT-S Int. Microw. Symp. (IMS), June 2019, pp. 472-475.

[26] S. Lee et al., "Digital predistortion for power amplifiers in hybrid MIMO systems with antenna subarrays," in Proc. IEEE 81st Veh. Tech. Conf. (VTC), 2015, pp. 1-5.

[27] L. Liu, W. Chen, L. Ma, and H. Sun, "Single-PA-feedback digital predistortion for beamforming MIMO transmitter," in Proc. IEEE Int. Conf. Microw. Millim. Wave Tech. (ICMMT), vol. 2, 2016, pp. 573-575.

[28] X. Liu, et al., "Beam-oriented digital predistortion for 5G massive MIMO hybrid beamforming transmitters," IEEE Trans. Microw. Theory Techn., vol. 66, no. 7, pp. 3419-3432, 2018.

[29] E. Ng, Y. Beltagy, G. Scarlato, A. B. Ayed, P. Mitran, and S. Boumaiza, "Digital predistortion of millimeter-wave RF beamforming arrays using low number of steering angle-dependent coefficient sets," IEEE Trans. Microw. Theory Techn., vol. 67, no. 11, pp. 4479-4492, 2019.

[30] E. Ng, Y. Beltagy, P. Mitran, and S. Boumaiza, "Single-input singleoutput digital predistortion of power amplifier arrays in millimeter wave RF beamforming transmitters," in Proc. IEEE/MTT-S Int. Microw. Symp.-IMS, 2018, pp. 481-484.

[31] C. Fager, T. Eriksson, F. Barradas, K. Hausmair, T. Cunha, and J. C. Pedro, "Linearity and efficiency in 5G transmitters: New techniques for analyzing efficiency, linearity, and linearization in a $5 \mathrm{G}$ active antenna transmitter context," IEEE Microw. Mag., vol. 20, no. 5, pp. 35-49, 2019.

[32] D. Pozar and D. Schaubert, "Scan blindness in infinite phased arrays of printed dipoles," IEEE Trans. Antennas Propag., vol. 32, no. 6, pp. 602-610, 1984

[33] D. M. Pozar, "The active element pattern," IEEE Trans. Antennas Propag., vol. 42, no. 8, pp. 1176-1178, 1994. 


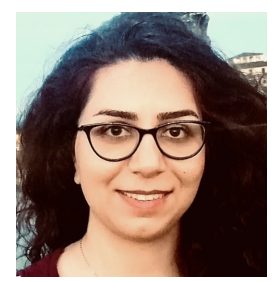

Parastoo Taghikhani received B.Sc and M.Sc degrees in electrical engineering, telecommunication from the Shahed University, Tehran, Iran in 2008 and 2011 respectively. She is currently pursuing a $\mathrm{PhD}$ degree at the Department of Microtechnology and Nanoscience (MC2), Chalmers University of Technology, Göteborg, Sweden. Her current research interests include modeling of active antenna arrays for mmWave MIMO transmitters. It also includes thermal modeling of active circuits for electrothermal and multiphysics analysis.

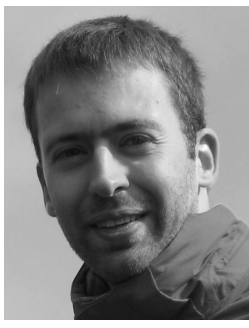

koen Buisman (S'05-M'09-SM'18) received the M.Sc. and Ph.D. degrees in microelectronics from the Delft University of Technology, Delft, The Netherlands, in 2004 and 2011, respectively. From 2004 to 2014, he was with the Delft Institute of Microsystems and Nanoelectronics, Delft University of Technology. In 2014, he joined the Chalmers University of Technology, Gothenburg, Sweden, where he is currently an Assistant Professor with the Microwave Electronics Laboratory, Department of Microtechnology and Nanoscience. He has authored or coauthored more than 70 refereed journal and conference articles. He holds one patent. His current research interests include nonlinear device characterization, technology optimization, and design of linear receivers for wireless systems.

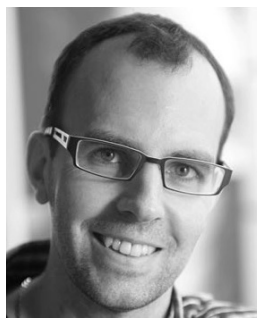

Christian Fager (S'98-M'03-SM'15) received his $\mathrm{Ph} . \mathrm{D}$. degree from Chalmers University of Technology, Sweden, in 2003. He is currently a Full Professor at Chalmers University of Technology and Head of the Microwave Electronics Laboratory. He has authored or coauthored more than 140 publications in international journals and conferences. His current research interest includes energy efficient and linear transmitters for future wireless communication systems.

Dr. Fager serves as Associate Editor of IEEE Microwave Magazine and IEEE Microwave and Wireless Components Letters. $\mathrm{He}$ is representative for Sweden, Norway and Iceland in the European Microwave Association (EuMA). He serves as a TPC Member for the IEEE MTT-S International Microwave Symposium (IMS) and is also chair/cochair of the 2020/2021 IEEE Topical Conference on RF/microwave Power Amplifiers and TPC co-chair for the 2020 European Microwave Integrated Circuits Conference. He received Chalmers Supervisor of the Year Award in 2018 and the IEEE International Microwave Symposium Best Student Paper Award in 2002. 